\title{
Antibiotic usage in chronic rhinosinusitis: analysis of national primary care electronic health records*
}

\author{
Claire Hopkins', Elizabeth Williamson², Steve Morris³, Caroline S. Clarke4, $\quad$ Rhinology 57: 6, 420 - 429, 2019 \\ Mike Thomas ${ }^{5}$, Hannah Evans², Paul Little ${ }^{5}$, Valerie J. Lund ${ }^{6}$, Helen Blackshaw ${ }^{6}$, https://doi.org/10.4193/Rhin19.136 \\ Anne Schilder ${ }^{6}$, Carl Philpott ${ }^{7}$, James Carpenter ${ }^{1,9}$, and Spiros Denaxas ${ }^{2}$, \\ on behalf of the MACRO programme team \\ *Received for publication: \\ March 29, 2019 \\ Accepted: July 12, 2019 \\ 2 Department of Medical Statistics, London School of Hygiene \& Tropical Medicine, London, UK \\ ${ }^{3}$ Department of Applied Health Research, University College London, London, UK \\ ${ }^{4}$ Department of Primary Care and Population Health, UCL, London, UK \\ ${ }^{5}$ University of Southampton, Southampton, UK \\ ${ }^{6}$ Ear Institute, UCL, London, UK \\ ${ }^{7}$ Norwich Medical School, University of East Anglia, Norwich, UK; ENT Department, James Paget University Hospital NHS Founda- \\ tion Trust, Great Yarmouth, UK \\ ${ }^{8}$ MRC Clinical Trials Unit at UCL, London, UK \\ ${ }^{9}$ Institute of Health Informatics, UCL, London, UK
}

\begin{abstract}
Background: The aim of this study was to analyse rates of antibiotic usage in chronic rhinosinusitis (CRS) in primary care in England and Wales and to identify trends in the choice of antibiotics prescribed.
\end{abstract}

Methods: We used linked data from primary care EHRs, with diagnoses coded using the Read terminology (Clinical Practice Research Datalink) from consenting general practices, with (2) hospital care administrative records (Hospital Episode Statistics, HES recorded using ICD-10).

Results: From the total of 88,317 cases of CRS identified, 40,462 (46\%) had an antibiotic prescription within 5 days of their first CRS diagnosis. Of patients receiving a first line antibiotic within 5 days of CRS diagnosis, over $80 \%$, in each CRS group, received a subsequent prescription for an antibiotic. Within 5 years of diagnosis, $9 \%$ are estimated to have had 5 or more antibiotics within 5 days of a CRS-related consultation. With data spanning almost 20 years, it was possible to discern trends in antibiotics prescriptions, with a clear increasing trend towards macrolide and tetracycline prescribing evident.

Conclusions: While antibiotics may have been prescribed for acute exacerbations, we have found high rates of repeated antibiotic prescription in some patients with CRS in primary care. There is a need for stronger evidence on the role of antibiotics in CRS management.

Key words: chronic rhinosinusitis, antibiotics

\section{Introduction}

Chronic rhinosinusitis (CRS) represents a common source of ill health; $11 \%$ of UK adults report CRS symptoms in a worldwide population study ${ }^{(1)}$. Symptoms, including nasal obstruction, nasal discharge, facial pain, anosmia and sleep disturbance, have a major impact on quality of life, reportedly greater in several domains of the SF-36 than angina or chronic respiratory disease (2). Acute exacerbations, inadequate symptom control and respiratory disease exacerbation are common problems faced by these patients ${ }^{(3,4)}$. 
CRS is a heterogeneous group of conditions, divided into two main phenotypic subgroups of those patients with and without nasal polyps. Unlike acute rhinosinusitis (ARS), CRS is not thought to have a primarily infectious aetiology; instead CRS is largely characterised by mucosal inflammation. Systemic antibiotics, therefore, probably have little role as antimicrobials in CRS management, with the exception of treating some acute exacerbations; NICE guidelines recommend withholding antibiotics in the majority of cases of uncomplicated ARS. The European Position Paper on Rhinosinusitis and Nasal Polyps (EPOS) published treatment guidelines and a research strategy for CRS, emphasising where limited evidence restricts care ${ }^{(5)}$, but there are currently no NICE guidelines for managing patients with CRS. Awareness and uptake of EPOS is variable, and both GPs and specialist ENT surgeons share uncertainty regarding the role of antibiotics ${ }^{(6)}$. A recent ENT-UK commissioning guideline ${ }^{(7)}$ acknowledges the lack of high quality trials and does not recommend routine antibiotic use for CRS in primary care.

There is growing interest in immune-modulating effects of macrolide antibiotics in chronic airway inflammatory disease, with a low-dose long term macrolide being prescribed for its immune response and not primarily as an anti-bacterial agent ${ }^{(8)}$. A 2016 Cochrane review by Head et al. compared systemic or topical antibiotic treatment to placebo, no treatment or other pharmacologic interventions, evaluated in RCTs with a follow-up period of at least three months. The authors concluded that there was very little evidence that systemic antibiotics are effective in patients with CRS. They found moderate quality evidence of a modest improvement in disease-specific quality of life in adults with CRS without polyps receiving three months of an oral macrolide, with a moderate improvement size $(0.5$ points on a $5 \square$ point scale) seen only at the end of the three-month treatment course of oral macrolides; by three months later, no difference was found ${ }^{(9)}$. The authors concluded that more research is needed, especially regarding longer-term outcomes and adverse effects. Longer-term antibiotic use in secondary care has a lowgrade recommendation in EPOS reflecting conflicting evidence from 2 RCTs ${ }^{(10,11)}$ resulting in a call for further trials ${ }^{(9,12-14)}$.

Despite this, CRS is said to account for $7.1 \%$ of primary care visits in the US where an antibiotic is prescribed ${ }^{(15)}$, making it the most common diagnosis associated with antibiotic usage in primary care. A 2011 U.S. study using electronic prescribing records found that primary care physicians prescribed antibiotics in $53.5 \%$ of over 36 million visits for CRS in a calendar year (16). A previous UK study evaluated antibiotic prescribing for 'rhinosinusitis' in primary care, with $91 \%$ receiving an antibiotic prescription ${ }^{(17)}$; however, this study did not differentiate between acute and chronic rhinosinusitis.
Antimicrobial stewardship describes measures aimed to improve the effectiveness and reduce the adverse effects of antimicrobial use, by reducing inappropriate antibiotic prescriptions, unsuitable use of broad-spectrum antibiotics and inappropriate dosage or duration use. This is driven by concerns of increasing antimicrobial resistance that is a major patient safety and public health concern as well as an economic issue ${ }^{(17,18)}$. Given the high prevalence of $C R S$, potentially high utilisation of antibiotics may represent a significant target for stewardship. The aim of this study was to analyse rates of antibiotic usage in CRS in England and Wales and to identify trends in the choice of antibiotics prescribed. This study is part of the wider MACRO programme "Defining best Management of Adult patients with Chronic RhinOsinusitis" that aims to define best practice for treating and managing patients with CRS and will include an RCT to generate more evidence regarding the use of macrolides.

\section{Materials and methods}

\section{Data sources}

We used linked data from 1) primary care Electronic Health Records (EHR), with diagnoses coded using the Read controlled clinical terminology (Clinical Practice Research Datalink, CPRD ${ }^{(19)}$ ) from consenting general practices, with 2) hospital care administrative records (Hospital Episode Statistics, HES diagnoses recorded using ICD-10 and procedures using OPCS-4). Phenotyping algorithms identifying disease status were created using the CALIBER approach described and validated elsewhere ${ }^{(19-21)}$. Data were extracted from the CPRD GOLD (January 2017 version).

\section{Eligibility}

The study period was 1st April 1997 to 29th February 2016. Male and female patients of 16 years of age and above, who were eligible for linkage to HES and ONS and had a minimum of one year of up-to-standard follow-up in CPRD prior to entry in the cohort were eligible for inclusion in our study (where "upto-standard" denotes a CPRD indication of research-quality data from the contributing general practice). Eligible patients were also required to have at least one day of up-to-standard information and data at an individual level deemed to be acceptable research quality by CPRD.

\section{Follow-up}

Follow-up began at the last of: the current registration date of the patient at their general practice, the up-to-standard date of the general practice or 1st April 1997, plus one year of up-tostandard follow-up data. If the patient's 16th birthday came later, follow-up began at their 16th birthday.

Follow-up ended at the first of: the date of the patient's transfer out of the general practice, the last collection data of the general practice, date of death (recorded in either CPRD or ONS) or 29th 
February 2016 (study end date chosen to align with the HES linkage censoring date).

\section{Case ascertainment}

An EHR phenotype comprising primary care and secondary care diagnoses, and secondary care procedures deemed to indicate a definite diagnosis of chronic rhinosinusitis (CRS) cases was developed (Appendix 1). Patients with one or more of these diagnoses or procedures recorded during follow-up were considered to be definite CRS cases, with the date of diagnosis taken to be the date of the first such diagnosis or procedure. These patients had received a diagnosis code specifying $C R S$, or had undergone surgery for CRS. An example is the ICD10 code J320 "chronic maxillary rhinosinusitis" being recorded as the diagnosis code during a consultation was deemed to indicate a definite case of CRS.

An EHR phenotype indicating primary or secondary care diagnosis terms and secondary care procedures deemed to indicate "very likely" or "likely" CRS diagnostic events was also developed (see appendix B). As an example, a recorded ICD-10 code of 243 "rhinosinusitis", where chronicity was not specified was taken to be a potential "likely" diagnostic event, as we were unable to discern if the GP felt the episode was acute or chronic.

Primary care symptoms were classified into four non-overlapping categories based on the European Position Paper on Rhinosinusitis and nasal Polyps (EPOS 2012) guidelines, representing: nasal obstruction, nasal discharge, change in sense of smell, and headache. A single primary care consultation in which the patient reported symptoms classed as either nasal obstruction or nasal discharge, and additionally reported symptoms classed in any of the three remaining symptom categories was taken to be a potential "very likely" CRS diagnostic event.

In order to meet the definition of CRS, where duration of disease must be at least 3 months, the following criteria were then applied;

Patients with no definite CRS diagnosis at any time during follow-up were deemed to be very likely CRS cases if they had two "very likely" CRS events between three and nine months apart during follow-up. The first two events satisfying this condition were identified and the date of very likely CRS diagnosis taken to be the later date of the two events.

Patients with no definite or very-likely CRS diagnosis at any time during follow-up were deemed to be likely CRS cases if they had either one "very likely" CRS diagnostic event and one "likely", or two "likely" diagnostic events, between three and nine months apart during follow-up. The first two events satisfying this condition were identified and the date of likely CRS diagnosis taken to be the later date of the two events.

Our resulting cohort contains all eligible patients who were classed as having a definite, very likely, or likely CRS diagnosis.

\section{Quantitative variables}

\section{Description of CRS cases}

CRS definite, very likely and likely cases were described, by certainty of CRS diagnosis, in terms of the age (at diagnosis), gender, socioeconomic status and geographical profile, and polyp status. Due to the limitations of the data, CRS cases were defined as "polyps present" or "polyps unknown", the former representing a group where the presence of nasal polyps was recorded at some point in the patient journey.

\section{Antibiotic prescribing}

"First-line" antibiotics were defined as any antibiotic prescribed on the day of CRS diagnosis, or within 5 days following diagnosis. Antibiotics were classed into: Cephalosporins, Metronidazole, Quinolones, Macrolides, Penicillins, Tetracyclines, or Other. Where multiple antibiotics were prescribed on the same day, these were classed as "multiple" antibiotics. Of patients prescribed first-line antibiotics, the proportion being prescribed each class was graphed by year of CRS diagnosis, overall, by certainty of CRS diagnosis, and by polyp status (ever during follow-up or not). The number of post-CRS diagnosis antibiotic prescriptions was tabulated overall, and by class of antibiotic. The indications for prescribing are not recorded specifically in EHR, and diagnosis codes are not as frequently recorded at follow-up consultations; for repeat prescriptions we therefore considered the following; any antibiotics prescribed in relation to any consultation (termed "for any indication"); any antibiotic prescribed within 5 days of a CRS-related consultation (termed "for CRS"). As follow-up differs for different patients, so these numbers merely reflect what is in our data, rather than estimating a quantity such as "average number of prescriptions over 10 years". Second-line antibiotics were classed as the first other antibiotic prescription at any point (which may be of the same class as the first-line antibiotic).

\section{Statistical analysis}

Descriptive statistics were used to characterise the data. The estimated probability $(95 \% \mathrm{Cl})$ of any antibiotic being given for any diagnosis within 2.5 years was calculated as well as the estimated probability $(95 \% \mathrm{Cl}$ ) of an antibiotic being given for CRS (defined as an antibiotic prescription issued within 5 days of a CRS-related consultation) within 2.5 years. Also the median time in months between the first and second antibiotic courses for any indication and for CRS was calculated with interquartile ranges ( 25 th -75 th percentile). 


\section{Ethics}

Access to Clinical Practice Research Datalink (CPRD) data was granted following an Independent Scientific Advisory Committee (ISAC) application; Protocol number 16_200R.

Table 1.1. CRS cases identified according to grouping.

$\begin{array}{ccc}\text { CRS diagnosis certainty } & \text { Frequency } & \% \\ \text { Definite } & 62,685 & 71.0 \\ \text { Very likely } & 5,883 & 6.7 \\ \text { Likely } & 19,749 & 22.4\end{array}$

\section{Results}

Identified CRS cases

From the total of 88,317 cases of CRS identified, $71 \%$ were classified as definite, with $6.7 \%$ very likely and $22.4 \%$ likely (Table 1.1). The details of the demographics are shown in Table 1.2 with a mean age of 51 and a slight female preponderance in the "definite" group.

\section{Descriptive data \\ Antibiotic usage}

Within the total cohort of CRS cases identified, 40,462 (46\%) had an antibiotic prescription within 5 days of their first CRS diagnosis (Table 2.1). This proportion varied considerably with

Table 1.2. Demographics by CRS grouping.

\begin{tabular}{|c|c|c|c|c|c|c|}
\hline & \multicolumn{2}{|c|}{ CRS definite $(n=62,685)$} & \multicolumn{2}{|c|}{ CRS very likely ( $n=5,883)$} & \multicolumn{2}{|c|}{ CRS likely ( $n=19,749)$} \\
\hline & Freq & $\%$ & Freq & $\%$ & Freq & $\%$ \\
\hline Age (yrs; mean (SD)) & 51.0 & $(16.2)$ & 49.3 & $(16.3)$ & 47.4 & $(15.3)$ \\
\hline \multicolumn{7}{|l|}{ Sex } \\
\hline Male & 29,306 & 46.8 & 1,451 & 24.7 & 4,292 & 21.7 \\
\hline Female & 33,379 & 53.2 & 4,432 & 75.3 & 15,457 & 78.3 \\
\hline \multicolumn{7}{|l|}{ Ethnicity (HES) } \\
\hline White & 49,725 & 79.3 & 1,073 & 18.2 & 3,105 & 15.7 \\
\hline Indian subcontinent & 1,114 & 1.8 & 21 & 0.4 & 67 & 0.3 \\
\hline Black & 642 & 1.0 & 7 & 0.1 & 19 & 0.1 \\
\hline China/SE Asia & 450 & 0.7 & 2 & 0.0 & 18 & 0.1 \\
\hline Mixed & 830 & 1.3 & 8 & 0.1 & 44 & 0.2 \\
\hline Unknown & 9,924 & 15.8 & 4,772 & 81.1 & 16,496 & 83.5 \\
\hline \multicolumn{7}{|l|}{ Index of Multiple Deprivation } \\
\hline 1 (least deprived) & 16,124 & 25.7 & 1,586 & 27.0 & 5,407 & 27.4 \\
\hline 2 & 14,529 & 23.2 & 1,253 & 21.3 & 4,647 & 23.5 \\
\hline 3 & 13,007 & 20.7 & 1,285 & 21.8 & 4,094 & 20.7 \\
\hline 4 & 10,761 & 17.2 & 930 & 15.8 & 3,239 & 16.4 \\
\hline 5 (most deprived) & 8,184 & 13.1 & 824 & 14.0 & 2,351 & 11.9 \\
\hline Missing & 80 & 0.1 & 5 & 0.1 & 11 & 0.1 \\
\hline \multicolumn{7}{|l|}{ Practice region } \\
\hline North East & 1,520 & 2.4 & 33 & 0.6 & 300 & 1.5 \\
\hline North West & 10,710 & 17.1 & 1,317 & 22.4 & 2,840 & 14.4 \\
\hline Yorkshire \& the Humber & 2,701 & 4.3 & 141 & 2.4 & 1,144 & 5.8 \\
\hline East Midlands & 1,774 & 2.8 & 238 & 4.0 & 694 & 3.5 \\
\hline West Midlands & 7,931 & 12.7 & 903 & 15.3 & 2,112 & 10.7 \\
\hline East of England & 6,927 & 11.1 & 1,329 & 22.6 & 2,282 & 11.6 \\
\hline South West & 7,575 & 12.1 & 534 & 9.1 & 2,433 & 12.3 \\
\hline South Central & 7,965 & 12.7 & 682 & 11.6 & 3,203 & 16.2 \\
\hline London & 7,851 & 12.5 & 399 & 6.8 & 2,054 & 10.4 \\
\hline South East & 7,731 & 12.3 & 307 & 5.2 & 2,687 & 13.6 \\
\hline Polyps known to be present & 23,960 & 38.2 & 133 & 2.3 & 303 & 1.5 \\
\hline $\begin{array}{l}\text { Post-CRS-diagnosis follow-up (yrs), median } \\
\text { (25th, 75th percentile) }\end{array}$ & 5.1 & $(2.3,9.2)$ & 6.3 & $(2.9,10.5)$ & 5.2 & $(2.4,9.1)$ \\
\hline
\end{tabular}


Table 2.1. First and second line antibiotic prescribing where "for CRS" refers to those prescribed within 5 days of a CRS-related consultation.

\begin{tabular}{|c|c|c|c|c|c|c|c|c|}
\hline & \multicolumn{2}{|c|}{ Definite $(n=62,685)$} & \multicolumn{2}{|c|}{ Very likely $(n=5,883)$} & \multicolumn{2}{|c|}{ Likely $(n=19,749)$} & \multicolumn{2}{|c|}{ All $(n=88,317)$} \\
\hline & Freq & $\%$ & Freq & $\%$ & Freq & $\%$ & Freq & $\%$ \\
\hline $\begin{array}{l}\text { Antibiotic prescribed within } 5 \text { days of } \\
\text { CRS diagnosis }\end{array}$ & 18,232 & 29.1 & 4,542 & 77.2 & 17,688 & 89.6 & 40,462 & 45.8 \\
\hline Subsequent antibiotic given & 15,242 & 83.6 & 4,080 & 89.8 & 15,627 & 88.3 & 34,949 & 86.4 \\
\hline $\begin{array}{l}\text { Estimated probability }(95 \% \mathrm{Cl}) \text { of any } \\
\text { antibiotic within } 2.5 \text { years }\end{array}$ & & $\begin{array}{c}80.0 \\
(79.5,80.7)\end{array}$ & & $\begin{array}{c}88.0 \\
(86.9,89.0)\end{array}$ & & $\begin{array}{c}87.8 \\
(87.3,88.4)\end{array}$ & & $\begin{array}{c}84.4 \\
(84.0,84.7)\end{array}$ \\
\hline Subsequent antibiotic given for CRS & 8,957 & 49.1 & 2,990 & 65.8 & 10,843 & 61.3 & 22,790 & 56.3 \\
\hline $\begin{array}{l}\text { Estimated probability }(95 \% \mathrm{Cl}) \text { of an } \\
\text { antibiotic being prescribed for CRS } \\
\text { within } 2.5 \text { years }\end{array}$ & & $\begin{array}{c}42.3 \\
(41.5,43.1)\end{array}$ & & $\begin{array}{c}57.1 \\
(55.6,58.6)\end{array}$ & & $\begin{array}{l}54.5(53.7 \\
55.2)\end{array}$ & & $\begin{array}{c}49.3 \\
(48.8,49.8)\end{array}$ \\
\hline \multicolumn{9}{|c|}{ Time in months between first and second antibiotic (median (25th - 75th percentile)) } \\
\hline Any second antibiotic & 5.4 & $(1.7,14.2)$ & 4.4 & $(1.6,11.1)$ & 4.8 & $(1.6,11.3)$ & 5.0 & $(1.6,12.4)$ \\
\hline Second antibiotic for CRS & 9.4 & $(2.7,24.5)$ & 9.2 & $(3.0,22.8)$ & 9.9 & $(3.4,23.5)$ & 9.6 & $(3.0,23.8)$ \\
\hline
\end{tabular}

Table 2.2. Total number of antibiotic prescribing episodes by CRS group.

\section{Definite $(n=62,685) \quad$ Very likely $(n=5,883)$}

Likely $(n=19,749)$

All $(n=88,317)$

Overall total number of antibiotic prescribing episodes:

\begin{tabular}{|c|c|c|c|c|c|c|c|c|}
\hline 0 & 10,979 & 17.5 & 239 & 4.1 & 370 & 1.9 & 11,588 & 13.1 \\
\hline 1 & 9,237 & 14.7 & 597 & 10.1 & 2,342 & 11.9 & 12,176 & 13.8 \\
\hline 2 & 6,717 & 10.7 & 519 & 8.8 & 2,005 & 10.2 & 9,241 & 10.5 \\
\hline 3 & 5,210 & 8.3 & 449 & 7.6 & 1,734 & 8.8 & 7,393 & 8.4 \\
\hline 4 & 4,234 & 6.8 & 396 & 6.7 & 1,461 & 7.4 & 6,091 & 6.9 \\
\hline 5 & 3,332 & 5.3 & 344 & 5.8 & 1,304 & 6.6 & 4,980 & 5.6 \\
\hline$>5$ & 22,976 & 36.7 & 3,339 & 56.8 & 10,533 & 53.3 & 36,848 & 41.7 \\
\hline \multicolumn{9}{|c|}{ Total number of antibiotic prescribing episodes for CRS: } \\
\hline 0 & 30,536 & 48.7 & 816 & 13.9 & 1,151 & 5.8 & 32,503 & 36.8 \\
\hline 1 & 15,884 & 25.3 & 1,777 & 30.2 & 7,228 & 36.6 & 24,889 & 28.2 \\
\hline 2 & 6,196 & 9.9 & 974 & 16.6 & 3,911 & 19.8 & 11,081 & 12.5 \\
\hline 3 & 3,126 & 5.0 & 585 & 9.9 & 2,368 & 12.0 & 6,079 & 6.9 \\
\hline 4 & 1,817 & 2.9 & 435 & 7.4 & 1,480 & 7.5 & 3,732 & 4.2 \\
\hline 5 & 1,228 & 2.0 & 299 & 5.1 & 1,006 & 5.1 & 2,533 & 2.9 \\
\hline$>5$ & 3,898 & 6.2 & 997 & 16.9 & 2,605 & 13.2 & 7,500 & 8.5 \\
\hline
\end{tabular}

the certainty of CRS diagnosis, with $29 \%$ of definite CRS cases receiving an antibiotic within 5 days of diagnosis, compared with $90 \%$ of likely cases.

Of patients receiving a first line antibiotic within 5 days of CRS diagnosis, over $80 \%$ in each CRS group, received a subsequent prescription for an antibiotic, of any type, for any indication (Table 2.2). This was not restricted to being within a certain time from the first prescription. For those patients who received a second antibiotic, the median time gap between the first and second prescriptions was 5.0 months overall, with the interquartile range varying from 1.6 to 12.4 months. When restricted to repeat prescriptions for CRS only, $56.3 \%$ of cases received a subsequent prescription for an antibiotic. Patients followed for longer were more likely to have a second prescription; this can be overcome by estimating the probability of a subsequent antibiotic prescription assuming each patient was followed for a fixed period of time. Within 2.5 years of CRS diagnosis, $84 \%$ were estimated to receive a subsequent prescription for any indication; $49 \%$ were estimated to receive a subsequent antibiotic prescription for CRS.

Approximately half of the CRS cases had no post-diagnosis antibiotic prescriptions, but $26 \%$ had more than 10 recorded prescriptions of antibiotics during follow-up, with some going 

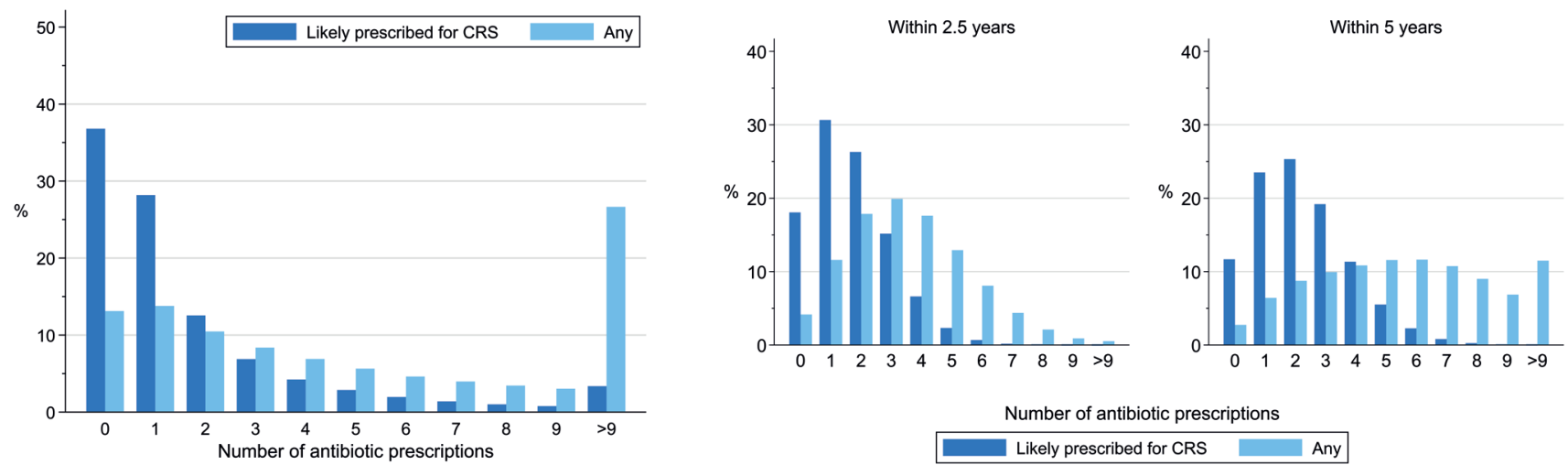

Figure 1. a) Overall number of antibiotic prescriptions following CRS diagnosis. b) Expected distribution of antibiotic prescriptions at 2.5 and 5 years post CRS diagnosis.
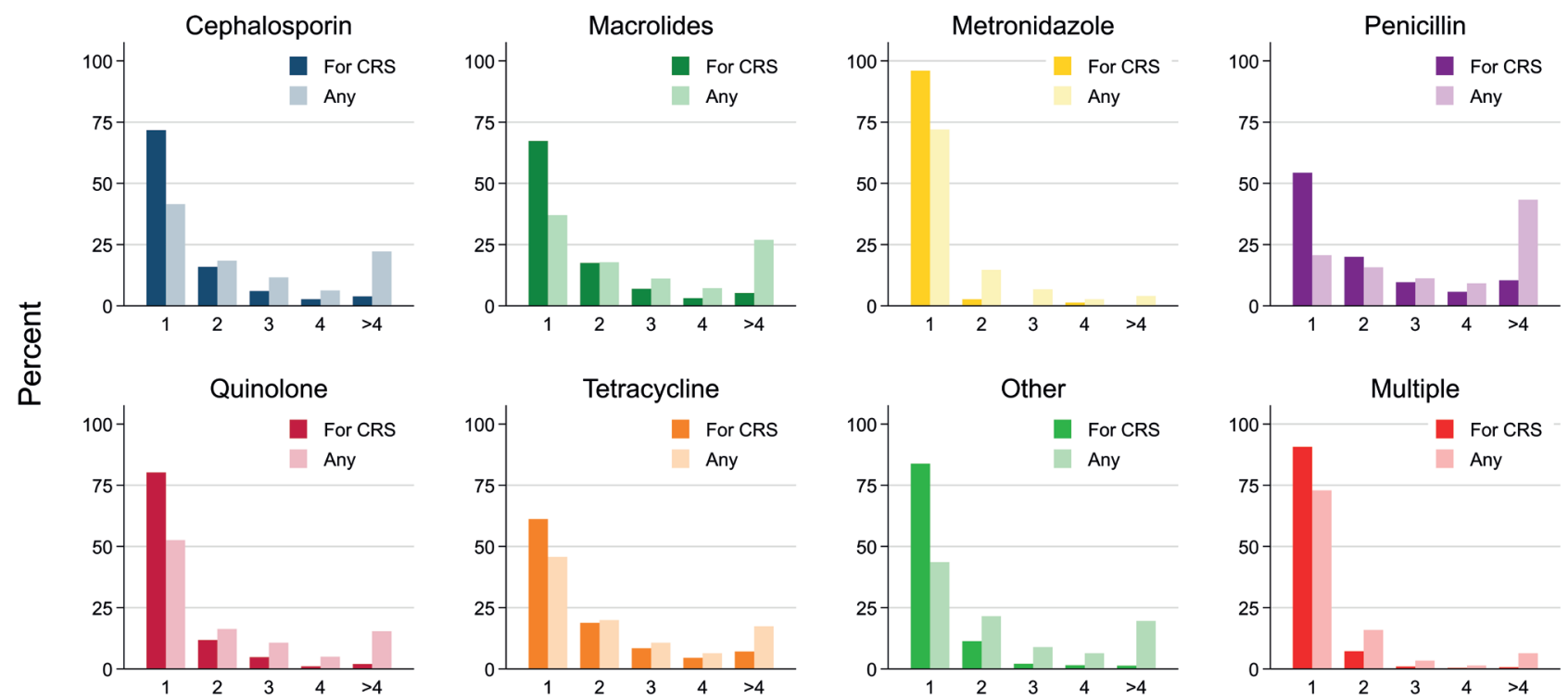

Number of prescriptions

Figure 2. Number of antibiotic prescriptions following CRS diagnosis, by class of initial first-line antibiotic.

into the hundreds (Table 2.1 and Figure 1a). Longer follow-up times are correlated with more prescriptions, ie patients with the high numbers of prescriptions may simply be a measure of the duration of patients' disease . Figure $1 \mathrm{~b}$ shows the expected distribution of numbers of antibiotic prescriptions within 2.5 years of diagnosis, and within 5 years, assuming full follow-up over that period. Within 2.5 years of CRS diagnosis, approximately $85 \%$ of cases are estimated to have had at least two antibiotic prescriptions; $50 \%$ of cases are estimated to have had at least two antibiotic prescriptions for CRS. Within 5 years of diagnosis, $61 \%$ of patients are estimated to have had 5 or more courses of antibiotics for any indication, and $9 \%$ are estimated to have had 5 or more antibiotics for CRS.

Figure 2 shows the number of antibiotic prescriptions during follow-up by class of the first antibiotic. So, for example, approximately $80 \%$ of patients prescribed penicillin as a first-line antibiotic were prescribed at least one additional course of penicillin during follow-up. Restricting the subsequent prescriptions to those within 5 days of a CRS-related consultation reduces this number to $45 \%$ of patients. For patients initially prescribed macrolides as a first-line antibiotic, approximately $25 \%$ had at least 5 prescriptions of macrolides during follow-up, $5 \%$ had at least 5 of those prescriptions within 5 days of a CRS-related consultation.

Figure 3 shows the duration of prescriptions. The most common recorded duration of both macrolide and penicillin prescriptions are 1 week, with lower peaks at 5 days, 2, 4, and 8 weeks. One or more macrolide courses of $8+$ weeks were received by 1,316 


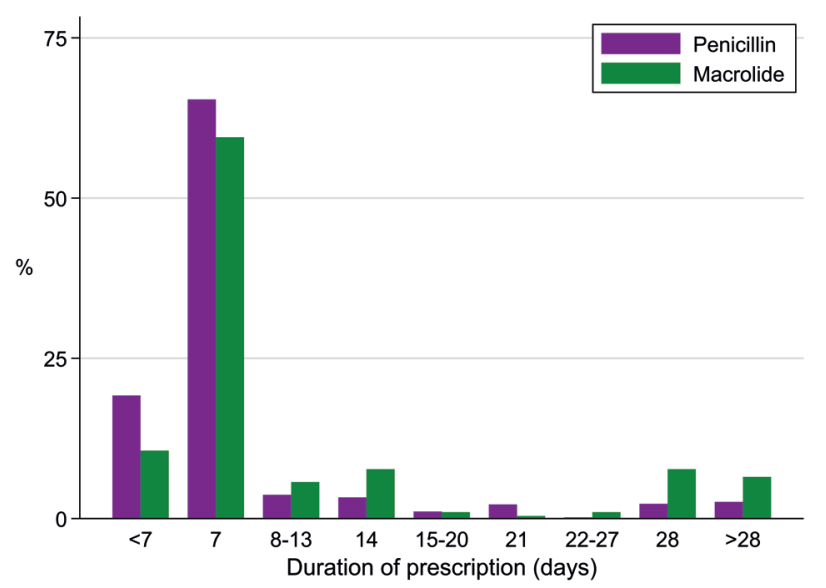

Figure 3. Duration of prescription given for macrolide and penicillin courses used in primary care among CRS definite cases following their CRS definite diagnosis.

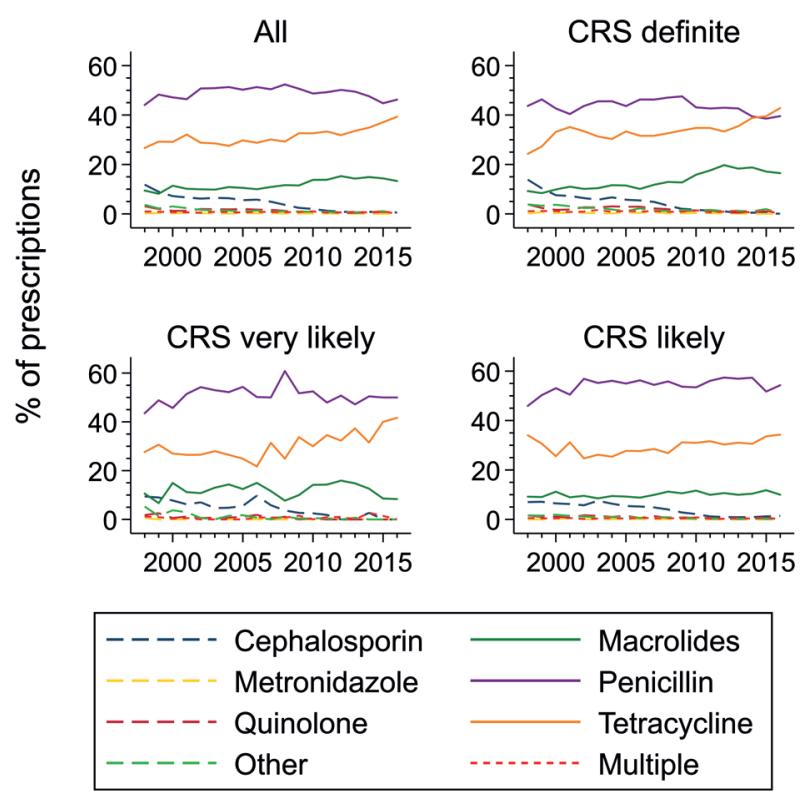

Figure 4. Trends in antibiotic prescribing by CRS diagnosis certainty.

cases (2.1\% of all CRS definite cases, $6.1 \%$ of those who received at least one macrolide prescription). One or more macrolides courses of 2 or more weeks were received by 6,269 cases $(9.9 \%$ of all CRS definite cases, $28.8 \%$ of those who received at least one macrolide prescription).

\section{Trends over time in antibiotic prescribing}

With EHR data spanning almost 20 years, it was possible to discern clear trends in antibiotics prescriptions. Throughout this period, penicillins remained the first choice of antibiotic even when macrolide and tetracycline prescribing became more common, when all likelihood of CRS diagnosis was considered, with approximately $50 \%$ of our CRS cohort receiving this. Howe-

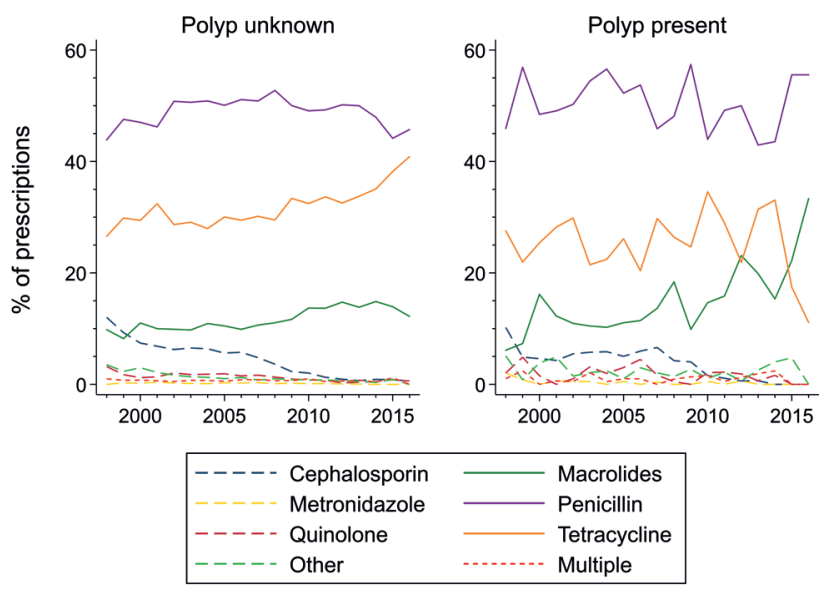

Figure 5. First-line antibiotic by polyp status.

ver, in those with definite CRS, a clear increasing trend towards macrolide and tetracycline prescribing was evident. It is likely that two key agents that account for this trend are doxycycline and clarithromycin. The data showed an increasing trend in macrolides as a first line prescription; rising from 9\% in 1998 up to $17 \%$ in 2015 (Figure 4). Similarly, prescriptions of tetracyclines increased from 24\% in 1998 to 43\%, and in 2015 tetracyclines were the most commonly prescribed antibiotic in CRS. In Figure 5 , the differences in prescribing habits for first antibiotic prescription can be seen for the two main assumed phenotypes of CRS. In those where polyps were seen, trends showed penicillin prescriptions to be steady with tetracyclines declining and macrolides increasing; the opposite was true in those where the polyp status was unknown.

\section{Discussion}

This is the first study evaluating antibiotic usage for chronic rhinosinusitis in primary care in a UK setting. In our study, 45\% of patients received antibiotics within 5 days of a consultation for CRS. The majority of patients in our cohort (71\%) had a definite diagnosis of CRS (i.e. a Read or ICD-10 code specific for chronic disease); in this group only $29 \%$ received antibiotics within 5 days of a consultation for CRS. This suggests that in the majority of patients where the diagnosis of chronic disease is clear, antibiotics are not being prescribed, in keeping with EPOS guidelines ${ }^{(5)}$. Similar interrogation of electronic health records in Germany found that $19.1 \%$ patients with a diagnostic code for CRS received an antibiotic prescription within 365 days of first diagnosis ${ }^{(22)}$. This reflects usage in our 'definite' cohort. Of those who have received an antibiotic, it is still possible that the antibiotics have been prescribed for acute infective exacerbations, which can be frequent in CRS; recent qualitative interviews with primary care physicians, antibiotics were prescribed for acute exacerbations, purulent discharge and occasionally in response to patient pressure if antibiotics have been helpful in previous 
episodes ${ }^{(6)}$.

The remainder had 'very likely' or 'likely' diagnoses of CRS (for example, 2 diagnoses of 'rhinosinusitis' more than 3 months apart but within 9 months). Our'very likely' and 'likely' cohorts include patients with 2 codes, more than 3 months apart, where the duration of sinusitis was not specified e.g. 'Rhinosinusitis' in contrast to CRS in our definite cohort. This may well reflect the uncertainty regarding chronicity of disease in the early stages; acute and chronic rhinosinusitis are differentiated in diagnostic criteria based on duration alone ${ }^{(5)}$ (i.e. less than or greater than 12 weeks) and share common symptoms of nasal blockage, nasal discharge, facial pain and pressure and change in sense of smell. Antibiotic usage increased in those with 'very likely' CRS (77\%) and with 'likely' CRS (89.5\%). This almost certainly reflects greater uncertainty regarding whether the episode was thought to be an acute or chronic presentation; nonetheless, this would still suggest very high levels of antibiotic usage even in ARS, given NICE recommendations against.

For patients who received an antibiotic at their first visit, more than $80 \%$ received a second course, with an average time of 5 months between courses. Within the whole CRS cohort, $26 \%$ of patients received more than 10 courses of antibiotics for any indication. Similar rates of repeat prescribing were demonstrated in all classes of antibiotics. Given the prevalence of CRS at $11 \%$ of UK adults, this suggests that, as in the US ${ }^{(16)}$, antibiotic prescribing rates for CRS represent a very large burden in primary care, and a significant target for antibiotic stewardship. The risks of unnecessary antibiotic use on resistance and the microbiome were highlighted in a recent EUFOREA brainstorming session to identify key research needs; it was also noted that one of the key challenges is that patients often expect antibiotics ${ }^{(23)}$.

Macrolide antibiotics were more likely to be prescribed for longer duration than penicillins; among all CRS cases, 1,323 (2.1\%) were prescribed at least one macrolide course of $\geq 8$ weeks and 6,269 (10.0\%) were prescribed a macrolide course of $\geq 2$ weeks. Restricting attention to the 21,549 CRS definite patients prescribed at least one macrolide, 1,323 (6.1\%) received at least one macrolide prescription of $\geq 8$ weeks and $6,269(29.1 \%)$ received at least one macrolide prescription of $\geq 2$ weeks. The UK Commissioning guidance for CRS advises against use of long term macrolides in primary care ${ }^{(7)}$. Contrary to this guidance, $12 \%$ of CCGs were found to make a recommendation to prescribe long term macrolide antibiotics to patients with symptoms suggestive of CRS prior to referral to secondary care ${ }^{(24)}$. The use of a prolonged course in primary care may therefore reflect local commissioning policies, although it may also occur on recommendation from secondary care after a more extensive investigation. We agree with the recommendation by Ferguson et al. ${ }^{(25)}$ that there should be a moratorium on the use of longterm courses of macrolide antibiotics in 'presumed' CRS, and use should be restricted to secondary care after confirmation of the diagnosis with objective findings such the endoscopic and/or radiological findings required by the EPOS guidelines ${ }^{(5)}$.

When looking at trends in first-line antibiotic choices over the last 20 years, overall rates of prescribing appear stable but there has been an increase in the use of both macrolides and tetracyclines (predominantly doxycycline), particularly in the CRS definite cases; although they appear to have been prescribed for the opposite CRS subgroup than recommended. NICE guidelines for ARS recommend penicillins as their first choice, and recommend doxycycline and macrolides only in cases of penicillin allergy. In secondary care, EPOS recommends for selected patients with CRS with nasal polyps, and as above, macrolides in selected patients with CRS without polyps and normal IgE levels $(5,26)$. The effectiveness of doxycycline has only been evaluated in one short-term $\mathrm{RCT}^{(27)}$ and a small open label study ${ }^{(28)}$ to date in patients with with polyps, showing moderate benefit in terms of reduction of polyp size compared with placebo, but in terms of symptom control was only found to reduce postnasal drip and not other nasal symptoms. Lack of access to endoscopy will severely restrict the ability to differentiate between CRS with and without polyps in primary care, and may account for the trend of increasing use of macrolides in patients with polyps and tetracyclines in CRS patients without polyps - in contradiction to recommended practice. In keeping with the principles of precision medicine, patients should be offered treatment tailored to their individual characteristics; this does not appear to be implemented successfully at present ${ }^{(29)}$.

The changes in patterns over time are likely to reflect trends in secondary care driving primary care prescribing habits. It is evident that both ENT and primary care doctors would benefit with support in applying evidence-based care in CRS, in addition to further research to establish the true role of antibiotics in the management of CRS. This observational study has been carried out as part of the MACRO Programme that involves a randomised controlled trial that will include a 12-week course of clarithromycin in patients with CRSwNPs and CRSsNPs.

\section{Limitations of this study}

We are likely to be underestimating overall antibiotic usage in CRS, as secondary care prescribing is not captured in our electronic health records, and may be as high as in primary care. The repeated use of antibiotics in some patients reflect the challenges in managing recalcitrant CRS patients ${ }^{(30)}$; these patients in particular may be receiving additional medical management, including antibiotics, in secondary care settings.

It is also possible that antibiotics have been prescribed for CRS 
without a diagnostic code being recorded, and therefore primary care prescribing likely lies between the rates of antibiotics linked within 5 days of a CRS code and for all diagnoses - we have therefore presented both rates in our analysis. We must rely on the diagnostic codes recorded and are unable to verify the diagnosis of CRS in any of our patients with endoscopy or CT scanning. The majority of patients in our cohort had 'definite' CRS; however, they may have presented with an acute exacerbation. Our assumptions in creating our very likely and likely cohorts may be incorrect, and this group may include patients with ARS, allergic rhinitis and other related conditions.

\section{Conclusion}

Based on current evidence and guidelines, antibiotics are not routinely recommended for the treatment of CRS, and even in the setting of acute exacerbations, their value remains unclear In this study we have found high levels of antibiotic prescribing in patients with 'likely' and 'very likely' CRS in primary care; although this probably reflects the diagnostic uncertainty around CRS in that setting and may represent a response to assumed acute exacerbation. While the majority of patients with a definite diagnosis of CRS did not receive an antibiotic prescription, there remains a high level of repeat antibiotic prescribing; in particular, nearly 1 in 10 patients received more than 5 prescriptions for antibiotics for CRS. Nearly 1 in 2 patients who received a macrolide antibiotic prescription were given a prolonged course of greater than 2 weeks. Use of antibiotics in patients with CRS in primary care appears to be an important potential target for antimicrobial stewardship that has been so far overlooked.

\section{Acknowledgements}

This paper presents independent research funded by the National Institute for Health Research (NIHR) under its Programme Grants for Applied Research (PGfAR) Programme (Grant Reference Number RP-PG-0614-20011). The views expressed are those of the authors and not necessarily those of the NHS, the NIHR or the Department of Health.

\section{Authorship contribution}

$\mathrm{CH}, \mathrm{CC}, \mathrm{MT}, \mathrm{PL}, \mathrm{VL}, \mathrm{HB}$ AS CP JC SD - design of study, interpretation of data and editorial review; $\mathrm{CH}$ EW, HE SD data analysis; $\mathrm{CH}$ drafting of manuscript

\section{Conflict of interest}

No conflicts of interest exists.

\section{References}

1. Hastan D, Fokkens WJ, Bachert C, Newson RB, Bislimovska J, Bockelbrink A, et al. Chronic rhinosinusitis in Europe--an underestimated disease. A GA(2)LEN study. Allergy. 2011;66(9):1216-23.

2. Gliklich RE, Metson R. The health impact of chronic sinusitis in patients seeking otolaryngologic care. Otolaryngol Head Neck Surg. 1995;113(1):104-9.

3. Erskine SE, Notley C, Wilson AM, Philpott CM. Managing chronic rhinosinusitis and respiratory disease: a qualitative study of triggers and interactions. J Asthma. 2015:52(6):600-5.

4. Erskine SE, Verkerk MM, Notley C, Williamson IG, Philpott CM. Chronic rhinosinusitis: patient experiences of primary and secondary care - a qualitative study. Clin Otolaryngol. 2016;41(1):8-14.

5. Fokkens WJ, Lund VJ, Mullol J, Bachert C, Alobid I, Baroody F, et al. European Position Paper on Rhinosinusitis and Nasal Polyps 2012. Rhinol Suppl. 2012(23):3 p preceding table of contents, 1-298.

6. Vennik J, Eyles C, Thomas M, Hopkins C Little P, Blackshaw H, et al. Management strategies for chronic rhinosinusitis: a qualitative study of GP and ENT specialist views of current practice in the UK. BMJ Open. 2018;8(12):e022643

7. Hopkins C, Philpott C, Carrie S, Blythe J, Thomas DM, Little P, et al. Commissioning Guide: Rhinosinusitis. London: ENT UK/ Royal College of Surgeons of England; 2016.
8. Cervin A, Wallwork B. Macrolide therapy of chronic rhinosinusitis. Rhinology. 2007:45(4):259-67

9. Head K, Chong LY, Piromchai P, Hopkins C, Philpott C, Schilder AG, et al. Systemic and topical antibiotics for chronic rhinosinusitis. Cochrane Database Syst Rev. 2016:4:CD011994

10. Wallwork B, Coman W, Mackay-Sim A, Greiff $\mathrm{L}$, Cervin A. A double-blind, randomized, placebo-controlled trial of macrolide in the treatment of chronic rhinosinusitis. Laryngoscope. 2006;116(2):189-93.

11. Videler WJ, Badia L, Harvey RJ, Gane S, Georgalas C, van der Meulen FW, et al. Lack of efficacy of long-term, low-dose azithromycin in chronic rhinosinusitis: a randomized controlled trial. Allergy. 2011;66(11):1457-68.

12. Pynnonen MA, Venkatraman G, Davis GE. Macrolide therapy for chronic rhinosinusitis: a meta-analysis. Otolaryngol Head Neck Surg. 2013;148(3):366-73.

13. Cervin A, Wallwork B. Efficacy and safety of long-term antibiotics (macrolides) for the treatment of chronic rhinosinusitis. Current allergy and asthma reports. 2014;14(3):416.

14. Bewick J, Ahmed S, Carrie S, Hopkins C, Sama A, Sunkaraneni $V$, et al. The value of a feasibility study into long-term macrolide therapy in chronic rhinosinusitis. Clin Otolaryngol. 2017:42(1):131-8

15. Smith SS, Evans CT, Tan BK, Chandra RK, Smith SB, Kern RC. National burden of antibiotic use for adult rhinosinusitis. J Allergy
Clin Immun. 2013;132(5):1230-2.

16. Lee LN, Bhattacharyya N. Regional and specialty variations in the treatment of chronic rhinosinusitis. Laryngoscope. 2011;121(5):1092-7.

17. Gulliford MC, Dregan A, Moore MV, Ashworth M, Staa T, McCann G, et al. Continued high rates of antibiotic prescribing to adults with respiratory tract infection: survey of 568 UK general practices. BMJ Open. 2014;4(10):e006245.

18. Smith R, Coast J. The economic burden of antimicrobial resistance: Why it is more serious than current studies suggest.: London School of Hygiene and Tropical Medicine; 2012.

19. Herrett E, Gallagher AM, Bhaskaran K, Forbes $H$, Mathur R, van Staa T, et al. Data Resource Profile: Clinical Practice Research Datalink (CPRD). Int J Epidemiol. 2015:44(3):827-36.

20. Herrett E, Shah AD, Boggon R, Denaxas $S$, Smeeth $L$, van Staa $T$, et al. Completeness and diagnostic validity of recording acute myocardial infarction events in primary care, hospital care, disease registry, and national mortality records: cohort study. BMJ. 2013;346:f2350.

21. Morley KI, Wallace J, Denaxas SC, Hunter RJ, Patel RS, Perel P, et al. Defining disease phenotypes using national linked electronic health records: a case study of atrial fibrillation. PloS one. 2014;9(11):e110900.

22. Park JJH, Seidel DU, Bachert C, Dazert S, Kostev K. Medication use in patients 
with chronic rhinosinusitis in Germany a large retrospective patient-based study. Rhinology. 2019;57(2):94-100.

23. Hellings PW, Akdis CA, Bachert $C$, Bousquet $J$, Pugin B, Adriaensen G, et al. EUFOREA Rhinology Research Forum 2016: report of the brainstorming sessions on needs and priorities in rhinitis and rhinosinusitis. Rhinology. 2017:55(3):202-10.

24. Soni-Jaiswal A, Philpott C, Hopkins C. The impact of commissioning for rhinosinusitis in England. Clin Otolaryngol. 2015;40(6):639-45.

25. Ferguson BJ, Narita M, Yu VL, Wagener MM, Gwaltney JM, Jr. Prospective observational study of chronic rhinosinusitis: environmental triggers and antibiotic implications. Clin Infect Dis. 2012;54(1):62-8.

26. Fokkens W, Lund V, Mullol J, European Position Paper on R, Nasal Polyps g.
European position paper on rhinosinusitis and nasal polyps 2007. Rhinol Suppl. 2007;20:1-136.

27. Van Zele T, Gevaert P, Holtappels G, Beule A, Wormald PJ, Mayr S, et al. Oral steroids and doxycycline: two different approaches to treat nasal polyps. J Allergy Clin Immunol. 2010;125(5):1069-76 e4.

28. Pinto Bezerra Soter AC, Bezerra TF, Pezato $R$, Teles Abdo TR, Pilan RM, Pinna FR, et al. Prospective open-label evaluation of longterm low-dose doxycycline for difficult-totreat chronic rhinosinusitis with nasal polyps. Rhinology. 2017;55(2):175-80.

29. Vlastos I, Gkouskou K, Doulaptsi M, Karatzanis A, Prokopakis EP. Precision Medicine in Rhinosinusitis. Curr Allergy Asthma Rep. 2019;19(2):12.

30. Prokopakis EP, Vlastos IM, Ferguson BJ, Scadding G, Kawauchi H, Georgalas C, et al. SCUAD and chronic rhinosinusitis. Reinforcing hypothesis driven research in difficult cases. Rhinology. 2014;52(1):3-8.

Professor Claire Hopkins

ENT Department

Guy's Hospital

London SE1 9R

United Kingdom

E-mail: clairehopkins@yahoo.com

\section{Appendix}

Appendix A: Case ascertainment for chronic rhinosinusitis

This appendix describes the case ascertainment algorithm developed, using primary and secondary care diagnostic terms and secondary care procedures, to identify patients with chronic rhinosinusitis (CRS). Certainty of the diagnosis based on this algorithm was classed as "Definite", "Very likely" or "Likely". All cases, irrespective of certainty of the diagnosis, were included in the current analyses.

\section{A.1. Definite diagnoses}

A codelist comprising primary care and secondary care diagnoses, and secondary care procedures deemed to indicate a definite diagnosis of chronic rhinosinusitis (CRS) cases was developed (codelist B.1.1 Definite). Patients with one or more of these diagnoses or procedures recorded during follow-up were considered to be definite CRS cases, with the date of diagnosis taken to be the date of the first such diagnosis or procedure. These patients had received a diagnostic code specifying CRS, or had undergone surgery for CRS

\section{A.2. Very likely and likely diagnoses}

A codelist indicating primary or secondary care diagnostic terms and secondary care procedures deemed to indicate "very likely" or "likely" CRS diagnostic events was also developed. Primary care symptoms were classified into four non-overlapping categories based on the European Position Paper on Rhinosinusitis and nasal Polyps (EPOS 2012) guidelines, representing: nasal obstruction, nasal discharge, change in sense of smell, and headache. A single primary care consultation in which the patient reported symptoms classed as either nasal obstruction or nasal discharge, and additionally reported symptoms classed in any of the three remaining symptom categories was taken to be a potential "very likely" CRS diagnostic event. A diagnosis of rhinosinusitis, where chronicity was not specified was taken to be a potential "likely" diagnostic event.
Patients with no definite CRS diagnosis at any time during follow-up were deemed to be very likely CRS cases if they had two "very likely" CRS diagnostic events between 3 and 9 months apart during follow-up. The first two events satisfying this condition were identified and the date of very likely CRS diagnosis taken to be the later date of the two events.

Patients with no definite or very-likely CRS diagnosis at any time during follow-up were deemed to be likely CRS cases if they had either one "very likely" CRS diagnostic event and one "likely", or two "likely" diagnostic events, between 3 and 9 months apart during follow-up. The first two events satisfying this condition were identified and the date of very likely CRS diagnosis taken to be the later date of the two events.

\section{Appendix B: Codelists}

\section{B1. CRS diagnostic terms}

The following codelists were developed to identify patients with chronic rhinosinusitis:

B.1.1. Codelist: Definite diagnostic events

B.1.2. Codelist: Very likely diagnostic events

B.1.3. Codelist: Likely diagnostic events

B.1.4. Codelist: Symptoms used in determining likely and very likely diagnoses

\section{B2. Macrolide antibiotics and penicillin}

The following codelists were developed to identify prescriptions of macrolide antibiotics and penicillin:

B.2.1. Codelist: Macrolide antibiotics

B.2.2. Codelist: Penicillin

The following pages provide these codelists. 